France, Norway and the USA participating in an international consortium of agricultural cohorts (AGRICOH).

Methods Use of each active ingredient was estimated from self-report (USA) or crop production combined with crop pesticide exposure matrices (France and Norway). Multivariable Cox regression was used to estimate overall and age-stratified ( adjusted for exposure to other pesticides and other potential confounders. Cohort-specific estimates were combined using random effects meta-analysis.

Results Among 316,270 farmers (75\% male), 63\% had ever used at least one pesticide, and 91 incident Hodgkin lymphoma cases were diagnosed during follow-up from 1993 to 2011 (3,574,815 person-years). Risks were elevated in association with use of the herbicide dicamba (meta-HR $=1.63,95 \%$ CI: $0.83-3.22 ; 35$ exposed cases), DDT (meta-HR=1.79, 95\% CI: $0.73-4.37 ; 27$ exposed cases), and the synthetic pyrethroid insecticides deltamethrin (meta- $\mathrm{HR}=1.86$, 95\% CI: $0.76-$ $4.52 ; 25$ exposed cases) and esfenvalerate (meta-HR=1.86, 95\% CI: $0.78-4.43 ; 22$ exposed cases), though precision was low.

Conclusion This was the largest effort from prospective studies to evaluate associations between the use of specific pesticides and the risk of Hodgkin lymphoma. Nevertheless, analyses were relatively underpowered due to low numbers of exposed cases. Future studies should aim to include data on Hodgkin lymphoma incidence among younger farmers and strive to further refine exposure assessment methods.

\section{RF-289 COLLECTING REAL-TIME SELF-REPORTED INFORMATION ON INTERMITTENT AGRICULTURAL ACTIVITIES USING SMARTPHONES}

${ }^{1}$ Pabitra Josse, Sarah Locke, Laura Beane Freeman, Jonathan Hofmann, Heather Bowles, Jon Moon, Melissa Friesen. ' U.S. National Cancer Institute, United States

\subsection{6/OEM-2021-EPI.355}

Introduction/Objective Farming is a highly variable occupation, with many tasks and exposures, making exposure assessment for epidemiologic studies challenging. We developed and deployed a smartphone app to collect real-time information on intermittent agricultural activities to characterize farming task variability.

Methods We recruited 19 male Iowa farmers, age 50-60 years, to log their farming activities in the app on 24 randomly selected days over 6 months. We populated the app with 350 farming activities; 152 activities were also linked to contextual questions (e.g., pesticide application method, PPE use). We calculated descriptive statistics on the number of activities reported and their duration.

Results The farmers provided activity information for 283 days. The farmers submitted 1,331 activities, representing 124 unique farming tasks. The median duration of a logged day was 545 minutes (interquartile range, IQR: 431-698). The median number of tasks reported per farmer was 18 (IQR: 5-31), with a median of 4 activities per day (maximum 17). The median duration of activities was 63 minutes (IQR: 32-133). The three most frequently reported tasks were related to animal work (36\% of activities), transportation (12\%), and crops (10\%). The tasks with the longest daily duration were planting crops (median: 415 minutes), mixing/loading/applying pesticides (365 minutes), and loading corn (270 minutes). The shortest tasks (median duration 10 minutes), were fueling trucks, collecting/storing eggs, and tree work. Over 36\% of the submitted activities also included responses to contextual questions; these were most frequently about feeding animals (56\%), transportation (25\%), and mixing/loading/ applying pesticides (6\%).

Conclusion Our findings show that it is possible to collect real-time, intermittent activity information over the span of several months. We captured most of the farming day and, as expected, observed substantial heterogeneity in activities and their durations, highlighting the need for individual-level activity data when evaluating risks in farmers.

\section{RF-401 AGRICULTURAL EXPOSURES AND RISK OF LYMPHOPLASMACYTIC LYMPHOMA/WALDENSTRÖM'S MACROGLOBULINEMIA IN THE AGRICULTURE AND CANCER (AGRICAN) COHORT}

${ }^{1}$ Amandine Busson, Pierre Lebailly, Séverine Tual, Alain Monnereau, Isabelle Baldi, Matthieu Meryet-Figuière, Stephanie Perrier, Mathilde Boulanger. 'Institut National de la Santé et de la Recherche Médicale (INSERM), France

\subsection{6/OEM-2021-EPI.356}

Introduction Many studies and meta-analyses concluded that farmers have an excess risk of Non-Hodgkin's Lymphoma (NHL) overall but results remain scarce for rare subtypes, like Lymphoplasmocytic Lymphoma/Waldenström macroglobulinemia (LPL/WM). In the AGRICAN cohort, the incidence of LPL/MW was found significantly higher among farmers than in the general population.

Objective Our aim was to study the association of LPL/WM with some agricultural exposures, like specific crops and livestock, or related tasks.

Methods Our analysis included 155,192 individuals from the 181,842 affiliated to the social agricultural scheme enrolled in 2005-2007 in 11 French areas. Exposure was determined from the report of work on 13 different crops and 5 livestock, for which 2 to 5 specifics tasks (including duration and size information) were collected. Incident cases were identified by cross-linkage with population-based cancer registries. Associations with crops, animals and specific tasks were analyzed using Cox models with age as time scale and farmers who were not exposed to the crop/livestock of interest as the referent group.

Results From enrollment to 2015, 1,349 incident NHL cases were identified including $122 \mathrm{LPL} / \mathrm{WM}$ cases. Elevated LPL/ WM risks were observed in (i) users of pesticides on crops $(\mathrm{HR}=1.56,95 \% \mathrm{CI}=1.03-2.38)$, especially on grasslands $(\mathrm{HR}=1.85,95 \% \mathrm{CI}=1.01-3.38)$, wheat/barley $(\mathrm{HR}=1.98,95 \%$ $\mathrm{CI}=1.24-3.16)$, and corn $(\mathrm{HR}=2.21,95 \% \mathrm{CI}=1.41-3.47)$; (ii) hay-makers $\quad(\mathrm{HR}=1.66, \quad 95 \% \mathrm{CI}=1.02-2.71)$; (iii) sowers $(\mathrm{HR}=1.28,95 \% \mathrm{IC}=0.75-2.20)$, especially on wheat/barley: $(\mathrm{HR}=1.70, \quad 95 \% \mathrm{CI}=1.09-2.65), \quad$ corn $\quad(\mathrm{HR}=1.59, \quad 95 \%$ $\mathrm{CI}=0.997-2.551)$ and root $(\mathrm{HR}=1.52,95 \% \mathrm{IC}=0.93-2.50)$; (iv) cattle breeders $(\mathrm{HR}=1.92,95 \% \mathrm{CI}=1.00-3.70)$, particularly with care $(\mathrm{HR}=1.97,95 \% \mathrm{CI}=1.02-3.81)$. Inverse associations were observed in poultry breeders $(\mathrm{HR}=0.44,95 \% \mathrm{CI}=0.28$ $0.70)$, particularly with care $(\mathrm{HR}=0.52,95 \% \mathrm{CI}=0.32-0.85)$, and disinfection of livestock premises $(\mathrm{HR}=0.28, \quad 95 \%$ $\mathrm{CI}=0.11-0.68)$.

Conclusion Elevated LPL/WM risks were linked with use of pesticide, haymaking and sowing tasks on several crops. Furthermore, specific associations were observed with some 